\title{
Strategies for cervical cancer screening in the scope of the Brazilian Unified National Health System
}

\author{
A.A.S. Reis ${ }^{1,2,3}$, C.C.F. Araújo ${ }^{1,3}$, E.G. da Silva ${ }^{1}$, T.B. Alves ${ }^{1}$, \\ Y.M.F. Ternes ${ }^{4}$ and R.S. Santos ${ }^{1,2}$ \\ ${ }^{1}$ Laboratório de Patologia Molecular, Instituto de Ciências Biológicas, \\ Universidade Federal de Goiás, Goiânia, GO, Brasil \\ ${ }^{2}$ Departamento de Bioquímica e Biologia Molecular, Instituto de Ciências \\ Biológicas, Universidade Federal de Goiás, Goiânia, GO, Brasil \\ ${ }^{3}$ Programa de Pós-Graduação em Ciências da Saúde, Faculdade de \\ Medicina, Universidade Federal de Goiás, Goiânia, GO, Brasil \\ ${ }^{4}$ Departamento de Saúde Coletiva, Instituto de Patologia Tropical e Saúde \\ Pública, Universidade Federal de Goiás, Goiânia, GO, Brasil
}

Corresponding author: A.A.S. Reis

E-mail: angela@ufg.br

Genet. Mol. Res. 20 (1): gmr18719

Received October 01, 2020

Accepted February 20, 2021

Published March 31, 2021

DOI http://dx.doi.org/10.4238/gmr18719

\begin{abstract}
Cervical cancer (CC) is the third most diagnosed malignancy among women in Latin America. CC control focuses on prevention methods: primary prevention is carried out to prevent Human Papillomavirus (HPV) infections, such as vaccination, while secondary prevention methods aim to detect and treat cervical premalignant lesions. In addition, cytology is used for triage, and many countries, including Brazil, still use it for this purpose. However, this method has significant limitations, such as low sensitivity and poor reproducibility. Some molecular assays are relevant, such as HPV testing. In this pilot study, a micro-costing analysis was done to compare cytology and HPV testing for cervical cancer screening in the Brazilian Unified Health System. For this assessment, a cross-sectional study was performed involving 500 women aged 18-55 years old without cervical precancer or cancer. The participants underwent cervical cytology and HPV testing. The overall prevalence of HPV infection was $25.2 \%$. Among these HPV-infected women, $13.6 \%$ presented normal or inflammatory, cytology and were
\end{abstract}


HPV positive by molecular assay. Most of these patients were infected with high-risk-HPV 16 and 18 and were asymptomatic. Oncotic cytology was the most cost-effective in the comparison between these two screening strategies. However, the molecular assay had a higher sensitivity and specificity when compared with cytology. HPV testing, despite the higher costs, may still be economically advantageous for CC control programs as it could reduce the cost of $\mathrm{CC}$ treatment in the public health system. In conclusion, although HPV testing is currently more expensive than cytology assay, its use would still be justified as it can reduce the economic and social impact due to cases not detected by cytology.

Key words: Brazilian Unified National Health System; Cervical cancer; HPV infection

\section{INTRODUCTION}

Cervical cancer (CC) is still a major public health challenge in low and middleincome countries. In Brazil, 16,360 new cases were estimated in 2018, making CC the third most frequent cancer type among women (Fabri et al., 2019). CC control includes primary prevention through vaccination to prevent Human Papillomavirus (HPV) infection, and secondary prevention through screening to detect and treat cervical precancerous lesions. Several screening methods that detect HPV have emerged in recent years. For instance, commercial assays were developed to diagnose cervical infections linked to high-risk HPVs (HR-HPVs) infection (Dijkstra et al., 2014). These assays are relevant because can identify HR-HPVs infection in cervical cancer precursor lesions (El-Zein et al., 2016).

In the primary health care network, the Brazilian National Program for Control of Cervical Cancer employs prevention actions including vaccination and early detection of CC guided by the Brazilian Guidelines for CC Screening (Possati-Resende et al., 2018). Although this program is comprised of a complex network responsible for the promotion and protection of health in Brazil, CC is still the second most frequent tumor type among women (Fabri et al., 2019). This cross-sectional study was designed to perform a microcosting analysis of available assays for the screening of CC in the Brazilian Unified Health System.

\section{MATERIAL AND METHODS}

The cross-sectional study population included 500 women randomized aged 18-55 years old without cervical precancer or cancer were evaluated during one year, who were attended gynecology professionals at Women's Hospital, Goiânia, Goiás, Brazil. Among the inclusion criteria were women over 18 years of age. Pregnant women, under 18 years of age, and cervical precancer and cancer, or another disease were considered exclusion criteria.

The study was conducted according to the Ethical Principles of the Declaration of Helsinki, a statement of ethical principles to guide physicians and other participants in medical research involving human subjects and approved by the ethics committee of the 
Federal University of Goiás under protocol number CAAE: 65007217.0.0000.5083. Written informed consent was obtained from all participants.

A micro-costing analysis was done to evaluate HPV testing as a tool to prevent cervical cancer for the Brazilian Unified Health System. For this, the participants underwent a cervical cytology test, and HPV testing via a molecular assay. Each participant underwent a pelvic exam to collect two cervical samples separately for cytology analysis and HPV testing by polymerase chain reaction (PCR). Outcomes were obtained according to triage: (1) cytology analysis, (2) HPV testing, (3) positive results in HPV testing were evaluated by genotyping HPV $6,11,16,31,33,35,45,56$, and 58. Additionally, lifestyle and demographic data were obtained via questionnaires.

Cervical brush samples were collected and preserved in Phosphate Buffered Saline solution. DNA extraction was performed using a commercial kit following the suggested

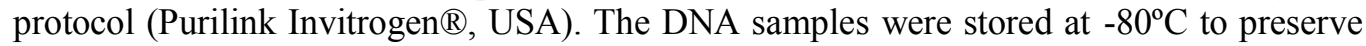
the characteristics of the nucleic acid for HPV testing. The samples were analyzed by PCR, using sets of primers, GP5+/GP6+ and subtype-specific primers for HPV subtypes 6, 11, 16, $31,33,35,45,56$, and 58 , respectively. The primers and thermocycling conditions PCR for were applied as previously described Chaiwongkot et al., (2007).

The Pap smears were examined by a Cytologist/Histopathologist using the Bethesda 2001 classification (Solomon et al., 2002). All slides were reviewed by two independent cytologists who were blinded to their counterpart's results. Confidentiality was assured by assigning a study code to each sample in both assays, and no confidential information was shared.

We measured the cost for both assays. Direct costs were estimated by microcosting, a technique that consists of a detailed enumeration of direct costs for every resource consumed separately for each diagnostic test execution. Micro-costing was estimated by the cost of every input reagent consumed for the molecular and cytology assays for each patient. Only operational costs were estimated, assuming that investments in infrastructure and equipment were unnecessary.

For this micro-costing analysis, the perspective of the analysis was the Brazilian public health system, and the outcome of interest was "cases diagnosed correctly". In the analysis three outcomes were possible: HPV infection correctly diagnosed (true positive), asymptomatic incorrectly diagnosed (false negative), and individual without HPV infection correctly diagnosed (true negative). To validate these assays (molecular and cytology), we used sensitivity and specificity criteria. We used the Cohen kappa index for agreement testing between the test results. The prices of the consumables were obtained from official sources. The costs of the assays were converted from Brazilian reals to US dollars at a rate of R $\$ 4.00=$ US $\$ 1.00$, which was the mean exchange rate at the time the data was collected. We used the chi-square test for categorical variables in the risk factors associated with HPV infection. A P-value of less than 0.05 was considered statistically significant. All statistical analyses were conducted using the STATA (v.13.0) program.

\section{RESULTS}

We evaluated 500 women using HPV testing and cytology for primary screening. The mean age of participants was $36( \pm 13.4)$ years old, and the age range with the highest number of HPV infections was 31 to 40 years old. Table 1 presents risk factors associated 
with HPV infection in the population studied. Of the women screened, 126 had a positive $\mathrm{HPV}$ test $(\mathrm{HPV}+)$, and 68 presented $\mathrm{HPV}+/$ cytology negative - demonstrating that they were asymptomatic for HPV infection $(54,0 \%)$.

Twenty women were previously reported as having HPV infections. However, we confirmed only seven as HPV+. The distribution of HPV types in these women was $42.8 \%$ for HPV 16, 28.6\% for HPV 18, 14.3\% for HPV 6, and 14.3\% for HPV 11. Three patients presented Cervical Intraepithelial Neoplasia 1 (CIN-1). Among the participants, 50.8\% reported having their first sexual intercourse at 16-20 years of age.

Table 1. Risk factors associated with Human Papillomavirus (HPV) infection.

\begin{tabular}{|c|c|c|c|}
\hline Characteristics & Total & HPV + (n/\%) & $p$ \\
\hline \multicolumn{4}{|l|}{ Age } \\
\hline$\geq 20$ & 54 & $17(31.5)$ & \multirow{4}{*}{$<0.00001^{*}$} \\
\hline $21-30$ & 130 & $23(17.7)$ & \\
\hline $31-40$ & 146 & $65(44.5)$ & \\
\hline$\geq 41$ & 170 & $21(12.3)$ & \\
\hline \multicolumn{4}{|c|}{ Number of sexual partners during life } \\
\hline 1 & 126 & $12(9.5)$ & \multirow{6}{*}{$<0.00001$} \\
\hline 2 to 3 & 180 & $53(29.4)$ & \\
\hline 4 to 5 & 66 & $27(40.9)$ & \\
\hline 6 to 10 & 28 & $22(78.6)$ & \\
\hline 11 to 20 & 22 & $7(3 ., 8)$ & \\
\hline$\geq 21$ & 78 & $5(6.4)$ & \\
\hline \multicolumn{4}{|l|}{ Smoking } \\
\hline Yes & 216 & $44(20.4)$ & \multirow{2}{*}{$0.030^{*}$} \\
\hline No & 284 & $82(28.9)$ & \\
\hline \multicolumn{4}{|c|}{ Alcohol consumption } \\
\hline Yes & 352 & $112(31.8)$ & \multirow{2}{*}{$<0.00001^{*}$} \\
\hline No & 148 & $14(9.4)$ & \\
\hline \multicolumn{4}{|c|}{ Oral contraceptive } \\
\hline Yes & 140 & $82(58.5)$ & \multirow{2}{*}{$<0.00001^{*}$} \\
\hline No & 360 & $44(12.2)$ & \\
\hline \multicolumn{4}{|l|}{ Pregnancy } \\
\hline None & 183 & $62(33.8)$ & \multirow{4}{*}{$<0.00001^{*}$} \\
\hline 1 to 2 & 236 & $32(13.5)$ & \\
\hline 3 to 4 & 59 & $17(28.8)$ & \\
\hline$\geq 5$ & 22 & $15(68.2)$ & \\
\hline \multicolumn{4}{|l|}{ Fixed partners } \\
\hline Yes & 260 & $54(20.7)$ & $0.001^{*}$ \\
\hline No & 240 & $72(30.0)$ & \\
\hline
\end{tabular}

Additionally, $56.9 \%$ (of the participants were non-smokers, and $70.4 \%$ considered themselves consumers of alcohol. Regarding the number of partners, 36\% reported having from two to three sexual partners throughout their lives. Regarding gynecology and obstetrics history, $28 \%$ reported using oral contraceptives and $47,2 \%$ having one or two children. Statistical analysis showed an association between risk factors with HPV infection (chi-square test, $\mathrm{P}<0.05$ ). None of the women underwent HPV vaccination. 
Table 2 displays frequencies of HPVs types by each oncotic cytology classification in the $126 \mathrm{HPV}+$ women. In cytological analyses, we did not observe cases harboring highgrade intraepithelial lesions (CIN). Atypical squamous cells of undetermined significance (ASCUS) were the most common finding, followed by low-grade CIN-1 and atypical squamous cells (ASCH). Most of these participants were infected with HR-HPVs 16 and 18.

Table 2. Distribution of Human Papillomavirus (HPV) types by HPV testing vs. cytology classification.

\begin{tabular}{lllllll}
\hline Cytology classification & $\begin{array}{l}\text { HPV }+ \\
\mathbf{n}(\%)\end{array}$ & $\begin{array}{l}\text { HPV 6 } \\
\mathbf{n}(\%)\end{array}$ & $\begin{array}{l}\text { HPV 11 } \\
\mathbf{n}(\mathbf{\%})\end{array}$ & $\begin{array}{l}\text { HPV 16 } \\
\text { n (\%) }\end{array}$ & $\begin{array}{l}\text { HPV 18 } \\
\text { n (\%) }\end{array}$ & $\begin{array}{l}\text { Other HPVs } \\
\text { n (\%) }\end{array}$ \\
\hline Normal or Inflammatory & $68(54,0)$ & $8(11.7)$ & $7(10.3)$ & $28(41.1)$ & $18(26.4)$ & $7(10.3)$ \\
ASCUS & $45(35,8)$ & $5(11.1)$ & $8(17.7)$ & $12(26.6)$ & $16(35.5)$ & $4(8.9)$ \\
ASCH & $6(4,7)$ & -- & -- & $3(50.0)$ & $2(33.3)$ & $1(16.6)$ \\
CIN - I & $7(5,5)$ & -- & -- & $4(57.1)$ & $2(28.5)$ & $1(14.3)$ \\
\hline Atypical Squamous Cells of Undetermined Significance (ASCUS), Atypical Squamous Cells (ASCH), Low-Grade Cervical Intraepithelial
\end{tabular}

Atypical Squamous Cells of Undetermined Significance (ASCUS), Atypical Squamous Cells (ASCH), Low-Grade Cervical Intraepithelial Neoplasia (CIN - I).

We observed that the oncotic cytology was the most cost-effective method among the two strategies analyzed (molecular test $=353.37$ vs. cytology $=63.02$ ) (Table 3). However, for a combination of both tests, there was an efficacy for HPV infection screening to detect and treat cervical precancer lesions. On the other hand, Kappa Index showed a low agreement between the molecular test results and oncotic cytology results, demonstrating that HPV testing had higher sensitivity and specificity than cytology $(\mathrm{KI}=0.08 ; \mathrm{P}=0.002)$. For cytology, the sensitivity was $68.25 \%$, and specificity was $100 \%$. Differentially, HPV testing presented $100 \%$ for these two variables.

Table 3. Cost-effectiveness analysis between the cytological and Human Papillomavirus (HPV) testing assays.

\begin{tabular}{lll}
\hline Method & Effectiveness & Cost-effectiveness \\
\hline Cytology & 0.172 & 63,02 \\
HPV testing & 0.252 & 353,37 \\
\hline$*$ Conversion rate used for the cytology and HPV testing assays: US\$1.00 $=\mathrm{R} \$ 4.00$.
\end{tabular}

\section{DISCUSSION}

In 2018, the World Health Organization (WHO) proposed actions to defeat CC based on proven strategies across the care continuum. Strategies included vaccination to prevent Human Papillomavirus (HPV) infection, screening, treatment of precancerous lesions, early detection, and prompt treatment of early invasive cancers (WHO, 2013; Bray et al., 2018; de Sanjose et al., 2019).

We observed that $25.2 \%$ of the 500 women presented HPV infection, and $13.6 \%$ of those infected were asymptomatic for viral infection. Additionally, cytology screening presents a particular set of issues. Specifically, this technique requires frequent examination and rigorous quality control to compensate for its low negative predictive value, lowmoderate sensitivity, and high variability depending on the operator (Gago et al., 2019). 
However, many countries, such as Brazil, use cytology for triage since it is one of the methods recommended by WHO guidelines (WHO, 2013).

Our findings demonstrated an association between risk factors and HPV infection $(\mathrm{P}<0.005)$. The age range with the highest percentage of HPV infection prevalence was $\geq 41$ years old, followed by $31-40$ years old $(\mathrm{P}<0.0001)$. The highest percentage of participants with HPV infection were women who reportedly have multiple sexual partners. These findings are similar to those of previous reports (Ma et al., 2017; del Arco et al., 2019). Sexual behaviors associated with HPV infections include multiple sexual partners, alcohol consumption, smoking, contraceptives use, not using a condom, and age differences between sexual partners (Herrera-Ortiz et al., 2018).

Our molecular analysis showed that HR-HPVs were the most prevalent among infected women, with the highest percentage of them having normal cytology. The distribution and prevalence of the HPV genotypes vary with age and geographical location (Chagas et al., 2015). In Brazil, HPV 16 is the most prevalent strain in high-grade lesions, whereas HPV 18 was found at a low frequency in abnormal smears (Britto et al., 2018). Some studies have shown that HPV16 is the most prevalent in Brazil (Gurgel et al., 2013; Freitas et al., 2014).

The prevalence of HR-HPVs 16 and 18 draws attention to the severity of the lesion being associated in approximately $70 \%$ of the $\mathrm{CC}$ cases worldwide but are also the most frequent types observed in women with normal cytology (Martins et al., 2016; Polman et al., 2017). However, most infected women will never develop cancer, because HPV infections are often transient and asymptomatic, and may regress between one and two years after exposure (de Farias et al., 2021).

ASCUS is the most common cervical abnormality in cytological results, followed by a low-grade squamous intraepithelial lesion (Arslan et al., 2018). In this pilot study, the findings demonstrated the overall prevalence for these histological types. The American Society for Colposcopy and Cervical Pathology offers three possible ways to manage ASCUS: follow-up with cytology, HPV testing, or colposcopy. Different countries choose distinct pathways depending on their resources, social factors, and health-care system features (Arslan et al., 2018).

Our results demonstrated that HPV testing was better than cytology at detecting HPV infection, although cytology was more cost-effective. HPV testing offers high sensitivity and negative predictive value, which allows extension of the screening interval (Gago et al., 2019), thus decreasing the risk for CC development. Further, it is critical to address the interpretation of HPV testing, recognizing that the positivity rate is higher than for cytology. However, cytology is commonly used in Brazil to find and treat cervical precancer to prevent CC (Possati-Resende et al., 2018).

Identifying and incorporating tools that complement both diagnosis and screening for CC precursor lesions is critical. Incorporating HPV testing will require in-depth analyses of the technical and operational aspects, and necessary resources to initiate and maintain such conduct without interruption. Law 12.401/2011 was a milestone in the Brazilian Unified Health System because it defines the criteria and timeframes for the health technology assessment process (Lima et al., 2019). Thus, incorporating new technologies such as HPV testing into the Brazilian Unified Health System is part of a rational process that evaluates technical, epidemiological, and financial criteria. 
In most countries utilizing this system and with ongoing quality assurance, cytology screening has led to significant reductions in CC incidence and mortality (de Sanjose et al., 2019). In Brazil, CC has a marked variation in incidence between the different regions of this vast country. Thus, utilizing HPV testing requires economic evaluation studies to quantify the costs and evaluate results following incorporation. Besides vaccinating and screening with cytology, HPV testing is an innovative approach in cervical precancer lesions screening programs, which can help overcome certain limitations of cytology-based methods (El-Zein et al., 2016).

\section{ACKNOWLEDGMENTS}

The authors would like to thank the Women's Hospital, Goiânia, Goiás, Brazil for providing samples for this study.

\section{CONFLICTS OF INTEREST}

The authors declare no conflict of interest.

\section{FUNDING STATEMENT}

This study was supported by public funding from Goiás State Foundation (FAPEG) and Research Program for the Brazilian Unified Health System (PPSUS) (grant number: 201410267000195 to A.A.S.R.).

\section{REFERENCES}

Arslan E, Gokdagli F, Bozdag H, Vatansever D, et al. (2018). Abnormal Pap-smear frequency and comparison of repeat cytological follow-up with colposcopy during patient management: The importance of pathologist's guidance in the management. North Clin. Istanbul. 14. 6(1): 69-74.

Bray F, Ferlay J, Soerjomataram I, Siegel RL, et al. (2018). Global cancer statistics 2018: GLOBOCAN estimates of incidence and mortality worldwide for 36 cancers in 185 countries. Cancer J. Clin. 6: 394-424

Britto AMA, Policarpo C, Pezzuto P, Meirelles ARI, et al. (2018). Detection of sexually transmitted infections at a Brazilian gynecology center: High prevalence of co-infections. J. Bras. Patol. Med. Lab. 54(6): 393-400.

Chagas BS, Comar M, Gurgel APAD, et al. (2015). Association study between cervical lesions and single or multiple vaccine-target and non-vaccine target human papillomavirus (HPV) types in women from northeastern Brazil. PLoS One. 10 (7): $\mathrm{e} 0132570$.

Chaiwongkot A, Pientong C, Ekalaksananan T, Kongyingyoes B, et al. (2007). Evaluation of primers and PCR performance on HPV DNA screening in normal and low grade abnormal cervical cells. Asian Pac. J. Cancer Prev. 8(2): 279-282.

De Farias FK, Silva ASG, dos Santos ACM, da Silva AF, et al. (2021). Human papillomavirus infection and risk factors to cervical cancer in asymptomatic women in the region of the Northeast of Brazil. J. Health Biol. Sci. 9(1): 1-6.

de Sanjose S and Holme F (2019). What is needed now for successful scale-up of screening? Papillomavirus Res. 16. 7: 173-5.

del Arco CD, Ayala BJ, García D, Sanabria C, et al. (2019). Distribution of cervical lesions in young and older women. Diagn Cytopathol. 47(7): 659-64.

Dijkstra MG, Snijders PJ, Arbyn M, Rijkaart DC, Berkhof J and Meijer CJ (2014).Cervical cancer screening: on the way to a shift from cytology to full molecular screening. Ann. Oncol. 25(5): 927-935.

El-Zein M, Richardson L and Franco EL (2016). Cervical Cancer Screening of HPV Vaccinated Populations: Cytology, Molecular Testing, Both or None. J. Clin. Virol. 76: S62-8.

Fabri VA, Queiroz ACM, Mantoan H, Sanches SM, et al. (2019). The impact of addition of consolidation chemotherapy to standard cisplatin-based chemoradiotherapy in uterine cervical cancer: Matter of distant relapse. J. Oncol. e1217838. 
Freitas LB, Chen Z, Muqui EF, Boldrini NAT, et al. (2014). Human papillomavirus 16 non-european variants are preferentially associated with high-grade cervical lesions. PLoS One. 9(7): e100746.

Gago J, Paolino M and Arrossi S (2019). Factors associated with low adherence to cervical cancer follow-up retest among HPV+/ cytology negative women: A study in programmatic context in a low-income population in Argentina. BMC Cancer. 19(1): e367.

Gurgel APAD, Chagas BS, Amaral CMM, Albuquerque EMB, et al. (2013). Prevalence and genetic variability in capsid L1 gene of rare human papillomaviruses (HPV) found in cervical lesions of women from North-East Brazil. Biomed. Res. Int. e546354.

Herrera-Ortiz A, Conde-Glez CJ, Olamendi-Portugal ML, García-Cisneros S, et al. (2018). College women, HPV genotyping and sexual behavior before HPV vaccination: Results from samples stored for a long time. J. Infect. Public Health. 11(2): 286-9.

Lima SGG, Brito C and Andrade CJC (2019). Health technology assessment in Brazil- an international perspective. Cien Saude Colet. 24(5): 1709-22.

Ma S, Stern JE, Feng Q, Hughes JP, et al. (2017). Incidence and risk factors for human papillomavirus infections in young female online daters. J. Med. Virol. 89(11): 2029-36.

Martins TR, de Oliveira CM, Rosa LR, Centrone CC, et al. (2016). HPV genotype distribution in Brazilian women with and without cervical lesions: Correlation to cytological data. Virol J. 13(1): 138.

Polman NJ, Veldhuijzen NJ, Heideman DAM, Snijders PJF, et al. (2017). HPV-positive women with normal cytology remain at increased risk of CIN3 after a negative repeat HPV test. Br. J. Cancer. 117(10): 1557-61.

Possati-Resende JC, Vazquez FL, Biot ST, Mauad EC, et al. (2018). Organized Cervical Cancer Screening Program in Barretos, Brazil: Experience in 18 Municipalities of São Paulo State. Acta Cytol. 62(1): 19-27.

Solomon D, Davey D, Kurman R, Moriarty A, et al. (2002). The 2001 Bethesda System: terminology for reporting results of cervical cytology. JAMA. 287(16): 2114-2119.

World Health Organization. Who Guidance Note. Comprehensive cervical cancer prevention and control: a healthier future for girls and women. 2013. Available at: [https://www.who.int/reproductivehealth/publications/cancers/9789241505147/en/] Accessed September 15, 2020. 